\title{
Klug handeln oder klug denken?
}

_ Die Zeiten ändern sich und mit ihnen ändern sich auch die Chirurgen. Die Zeit der „großen“ Schnitte als Qualitätsindikator für „große“ Chirurgen ist vorbei, so das Fazit des Deutschen Chirurgenkongresses. Klein und fein ist angesagt, so als hätte der Internist den Eingriff durchge-

führt. Auch wurde in

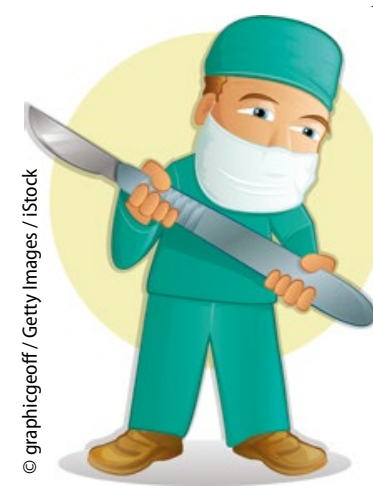
den letzten Jahren in der Chirurgie allmählich das Primat des Handelns ersetzt durch das Primat des Denkens. Dies ist

bei den Internisten schon lange Standard, z. B. wenn sie noch über EKG-Veränderungen disku- tieren, obwohl der Patient bereits tot ist. Somit ist den Chirurgen die akademische Emanzipation durchaus gelungen. Die Zeit ist vorbei, als der Internist dem Chirurgen anlässlich eines runden Geburtstags augenzwinkernd das Büchlein mit dem Titel „Die Kunst des klaren Denkens" schenken konnte und der Chirurg sich bei passender Gelegenheit revanchierte mit dem Buch „Die Kunst des klugen Handelns“.

Auch die phänotypische Abgrenzung des Chirurgen vom Internisten wird immer schwieriger, nachdem das Tragen einer Krawatte, über Jahrzehnte das Alleinstellungsmerkmal des Internisten, einem legeren Kleidungsstil wie ihn die Chirurgen bevorzugen, gewichen ist. Auch die Regelung, dass der Chirurg immer mit- ten durch den Gang stolzieren dürfe, während der Internist sich an der Wand entlang schleichen müsse, gibt es nicht mehr. Folgen sind vermehrt auftretende Kollisionen, was der viel gepriesenen interdisziplinären Zusammenarbeit sicherlich nicht förderlich ist.

Doch in einer konkreten Situation können Chirurg und Internist ihre ursprüngliche professionelle Prägung nicht überspielen, nämlich wenn es darum geht, die Lichtschranke an der Aufzugstür zu unterbrechen. Chirurgen setzen dabei weiterhin den Kopf ein, die Internisten nehmen die gefalteten Hände. Ein Schelm, wer böses dabei denkt!

Dr. Peter Stiefelhagen

\section{Chirurgen warnen}

\section{Vor Einnahme eines Bisphosphonats: Mundhöhle untersuchen!}

— „In Deutschland steigt die Zahl der Menschen, bei denen eine Therapie mit einem Bisphosphonat zu einer Osteonekrose des Kiefers geführt hat", stellte Prof. Michael Ehrenfeld, Präsident der Deutschen Gesellschaft für Mund-, Kiefer- und Gesichtschirurgie, fest. Auch wenn diese Substanzen in der Regel gut verträglich seien und ihr Nutzen für Osteoporosepatienten außer Zweifel stehe, müsse dieser Komplikation vermehrt Beachtung geschenkt werden.

Es beginnt mit Schmerzen und Schwellungen im Bereich des Kieferkammes. Daraus können sich Abszesse und Fisteln bilden, aus denen eitriges Sekret fließt. Die Röntgenaufnahme zeigt dann meist, dass bereits Teile des Kieferknochens abgestorben sind. „Die Fälle sind inzwischen so häufig, dass wir in München eine eigene Sprechstunde für solche Patienten eingerichtet haben“, so Ehrenfeld. Risikofaktoren sind Rauchen oder eine Therapie mit Steroiden oder Zytostatika. Betroffen sind vorwiegend ältere Patienten mit Parodontose oder Infektionen der Zahnwurzeln. „Wir vermuten, dass Entzündungen in der Nähe des Knochens die Toxizität der Bisphosphonate erhöhen", so Ehrenfeld.

Die Therapie ist langwierig. In Frühstadien kann der Krankheitsprozess durch Mundspülungen und Antibiotika gestoppt werden. In fortgeschrittenen Fällen ist eine Operation mit Ausräumung der Knochennekrosen unumgänglich. Dabei müssen oft Teile des Kiefers entfernt und durch ein Transplantat ersetzt werden. Wichtig ist die Prävention. „Die Bisphosphonat-Therapie sollte erst begonnen werden, wenn das Gebiss saniert ist, Zysten, desolate Zähne und Entzündungen im Mundraum müssen vorher beseitigt werden“, so der Gesichtschirurg.

- Deutscher Chirurgenkongress am 21.3.2017 in München 